\title{
Mecp2 Organizes Juvenile Social Behavior in a Sex-Specific Manner
}

\author{
Joseph R. Kurian, ${ }^{1}$ Meaghan E. Bychowski, ${ }^{2}$ Robin M. Forbes-Lorman, ${ }^{1}$ Catherine J. Auger,,${ }^{1,2}$ and Anthony P. Auger ${ }^{1,2}$ \\ ${ }^{1}$ Department of Psychology, and ${ }^{2}$ Neuroscience Training Program, University of Wisconsin-Madison, Madison, Wisconsin 53706
}

\begin{abstract}
Methyl-CpG-binding protein 2 (MeCP2) binds methylated DNA and recruits corepressor proteins to modify chromatin and alter gene transcription. Mutations of the MECP2 gene can cause Rett syndrome, whereas subtle reductions of MeCP2 expression may be associated with male-dominated social and neurodevelopmental disorders. We report that transiently decreased amygdala Mecp2 expression during a sensitive period of brain sexual differentiation disrupts the organization of sex differences in juvenile social play behavior. Interestingly, neonatal treatment with Mecp2 small interfering RNA within the developing amygdala reduced juvenile social play behavior in males but not females. Reduced Mecp2 expression did not change juvenile sociability or anxiety-like behavior, suggesting that this disruption is associated with subtle behavioral modification. This suggests that Mecp2 may have an overlooked role in the organization of sexually dimorphic behaviors and that male juvenile behavior is particularly sensitive to Mecp2 disruption during this period of development.
\end{abstract}

Key words: Mecp2; siRNA; amygdala; sex difference; behavior; epigenetic

\section{Introduction}

Methyl-CpG-binding protein 2 (MECP2) is an X-linked gene with a protein product $(\mathrm{MeCP} 2)$ that alters gene expression through several mechanisms. $\mathrm{MeCP} 2$ is primarily considered a methylated cytosine-binding protein that recruits chromatinmodifying factors, such as Sin3A and histone deacetylases, to repress gene transcription (Nan et al., 1998). More importantly, mutations of the MeCP2 protein cause Rett syndrome (RTT), a progressive neurological disorder occurring primarily in girls that is associated with loss of verbal language and motor control, such as stereotypical hand movements, and sometimes autisticlike behaviors (Amir et al., 1999; Samaco et al., 2005; Chahrour and Zoghbi, 2007). Because the MECP2 gene is located on the $\mathrm{X}$ chromosome, females with RTT can have a mosaic pattern of $\mathrm{MeCP} 2$ expression attributable to $\mathrm{X}$-inactivation. This results in varied expression of functional $\mathrm{MeCP} 2$ protein and a consequential continuum of phenotypic severity. In contrast, mutations in the coding region of the $M E C P 2$ gene are severe and usually lethal in males (Villard, 2007).

RTT is one of five related pervasive developmental disorders, which also include autism, Asperger's syndrome, childhood disintegrative disorder, and pervasive developmental disorder not otherwise specified. Of these disorders, only RTT has a known genetic cause with mutations in $\mathrm{MeCP} 2$ associated with the majority of RTT cases. However, not all MECP2 mutations result in

Received Dec. 7, 2007; revised May 28, 2008; accepted May 28, 2008.

This work was supported by National Institutes of Health (NIH) Grant R01MH072956.J.R.K. was supported by NIH Roadmap Interdisciplinary Award T32 MH75880. We thank Dr. Kris Olesen, Mira Kolodkin, and Jamie Kallan for technical assistance and Dr. Lauren Riters for assistance with statistical analyses.

Correspondence should be addressed to Anthony P. Auger, Department of Psychology, University of WisconsinMadison, 1202 West Johnson Street, Madison, WI 53706. E-mail: apauger@wisc.edu.

D0I:10.1523/JNEUROSCI.1345-08.2008

Copyright $\odot 2008$ Society for Neuroscience $\quad$ 0270-6474/08/287137-06\$15.00/0 classic RTT pathology but may be related to disorders with phenotypic similarity. For example, variations of a highly conserved region of the MECP2 3 ' -untranslated region, which is important for transcript stability, were found in autistic individuals (Shibayama et al., 2004). Significantly reduced MeCP2 expression was also found in frontal cortex samples of individuals with autism and attention deficit hyperactivity disorder (Nagarajan et al., 2006). Therefore, whereas mutations dramatically affecting MeCP2 activity cause RTT, it is possible that mutations or conditions that subtly alter levels of MeCP2 expression may be related to other neurodevelopmental disorders.

With emerging associations between reduced MeCP2 expression and male-dominated neurodevelopmental disorders, we recently questioned whether a sex difference in Mecp2 expression existed during brain development. We found that during rat development, males express less Mecp2 than females and that this sexual dimorphism exhibited spatial and temporal specificity (Kurian et al., 2007). Specifically, Mecp2 expression was lower in males on postnatal day 1 (P1) in the amygdala and ventromedial hypothalamus; but these differences no longer exist at postnatal day 10, a time point beyond the steroid-sensitive period of sexual differentiation of the rat brain. Furthermore, no expression differences were found at any time point or in any region tested for mbd2 (Methyl-CpG-binding domain protein 2), which has a function similar to Mecp2. Therefore, we predicted that Mecp2 had a specific role in sexual differentiation of the rat brain. Considering the regional specificity of the sex difference, we further predicted that sexually dimorphic behaviors, particularly juvenile social behavior organized during amygdala development, would be sensitive to disruption of Mecp 2 expression. Consequently, we examined the impact of brief Mecp2 disruption within the developing amygdala of males and females and determined the impact on social behavior development. 


\section{Materials and Methods}

Pup selection and preweaning environment. Adult female Sprague Dawley rats (Charles River Laboratories) were mated in our animal facility and allowed to deliver normally. Cages were checked regularly to determine the day of birth. On the day of birth, 60 pups were collected from five dams (12 from each dam consisting of six males and six females). Animals were separated into groups such that each dam contributed two pups to each small interfering RNA (siRNA) treatment group (male control, male Mecp2, female control, and female Mecp2) and four more pups (two male and two female) to a no treatment group. Therefore, experimental groups totaled 10 pups, evenly collected from the five dams. After the initial siRNA treatment, two pups from each pooled group were returned to one of the five dams so that each group was represented equally in each litter for the remainder of preweaning development. Pups receiving no treatment were included in preweaning litters and later participated in play groups and served as novel peer social stimulus animals in the three-chambered sociability test.

Mecp2 disruption with siRNA. Mecp2 (sc-35893; Santa Cruz Biotechnology) and control siRNA (sc-37007; Santa Cruz Biotechnology) were resuspended to $100 \mu \mathrm{M}$ in the supplied siRNA diluent with Lipofectamine LTX reagent (ratio of Lipofectamine to DNA was $1 \mu \mathrm{l}$ to 500 ng; Invitrogen) (Nagai et al., 2005). Infusions were done with a stereotaxic device modified to accommodate neonatal rats, and coordinates from center and bregma suture lines were empirically determined for direct infusion to the amygdala ( $1 \mathrm{~mm}$ lateral, $2 \mathrm{~mm}$ caudal, and $5.5 \mathrm{~mm}$ ventral from the pial surface at our nose cone angle). After cold anesthetization, rats were bilaterally infused with $1 \mu \mathrm{l}(100 \mathrm{nmol})$ of either Mecp2 or control siRNA and allowed to recover under a warm lamp before being returned to the dam. Each animal received three infusions $24 \mathrm{~h}$ apart on P0-P2. Animals were then allowed to develop typically with the dam until weaning at P21. This research was approved by the University of Wisconsin-Madison Animal Care and Use Committee.

Western immunoblotting. Snap-frozen microdissected amygdala and adjacent regions were homogenized after adding $300 \mu \mathrm{l}$ of ice-cold lysis buffer consisting of $50 \mathrm{~mm}$ Tris- $\mathrm{HCl}, 1 \% \mathrm{Na}$-deoxycholate, $0.25 \%$ Nonidet P-40, $150 \mathrm{~mm} \mathrm{NaCl}, 1 \mathrm{~mm}$ EDTA, Protease Inhibitor Cocktail (P8340, as directed; Sigma), and Phosphatase Inhibitor Cocktail (P2850, as directed; Sigma). To remove cellular debris and nuclei, the samples were centrifuged at $14,000 \mathrm{rpm}$ for $10 \mathrm{~min}$ at $4^{\circ} \mathrm{C}$. The supernatant was collected, and protein concentration was determined by a Lowery assay. Fifteen micrograms of total protein from each animal were gelelectrophoresed using a precast SDS-PAGE 4-20\% Tris-glycine gel (catalog \#58645; Cambrex Bio Science Rockland) and transferred to a polyvinyl difluoride membrane (Immobilon-P; catalog \#IPVH20200; Millipore). Membranes were blocked for $1 \mathrm{~h}$ in $0.1 \mathrm{~m} \mathrm{TBS}$ containing 5\% nonfat dry milk with constant agitation at room temperature. Membranes were then incubated overnight at $4^{\circ} \mathrm{C}$ with agitation in TBS containing $0.05 \%$ Tween-20 (TBS-T) and $2 \%$ nonfat dry milk with an antibody that recognizes the $\mathrm{C}$ terminus of human MeCP2 (amino acids 465-478), a sequence conserved in rat and mouse (1:2000 dilution; rabbit polyclonal; M9317; Sigma). The next day, the membranes were given three quick washes and then four 5 min washes in TBS-T. After being washed, membranes were incubated in a goat anti-rabbit horseradishperoxidase-linked secondary antibody (1:3000 dilution; catalog \#7074; Cell Signaling Technology) and horseradish-peroxidase-conjugated anti-biotin antibody (1:5000 dilution; catalog \#7075; Cell Signaling Technology) for $30 \mathrm{~min}$ at room temperature with agitation and washed three times for $5 \mathrm{~min}$ each with TBS-T and twice for $5 \mathrm{~min}$ each with TBS. Immunoreactive bands were detected using a chemiluminescence kit (catalog \#7003; Cell Signaling Technology) and exposed to x-ray film (catalog \#178 8207; Eastman Kodak). To correct for total protein concentration, membranes were incubated in Ponceau S solution (P7170; Sigma), which binds protein nonspecifically, for $5 \mathrm{~min}$ at room temperature with agitation and washed twice for 2 min each in distilled water. Ponceau S staining has been shown to correlate well with blotting for $\beta$-actin and allows for accurate quantification of the amount of total protein bound to a membrane (Klein et al., 1995). Because the protein band appearing at $\sim 45 \mathrm{kDa}$ contains all proteins potentially ranging from 43 to $47 \mathrm{kDa}$, normalizing to this band takes into account a multitude of proteins. The analysis of multiple proteins reduces the possibility of confounds attributable to sex differences in glyceraldehyde-3phosphate dehydrogenase (GAPDH) (Perrot-Sinal et al., 2001), actin (Stanley and Fink, 1986), or hormonal regulation of GAPDH (Funabashi et al., 1994) and $\beta$-actin (Matsumoto, 1997). Ponceau-S-stained membranes and the immunoblot films were digitized using a scanner and analyzed using the Western blot macro gel plot 2 within the Scion Image program. Ratios of Mecp2-immunoreactive protein to the nonspecific protein band were obtained for each animal and analyzed with a twotailed Student's $t$ test.

Behavioral testing and statistical analysis. All behavioral tests were performed under dim red light and began 1-2 $\mathrm{h}$ after the dark phase of the light cycle began. Each animal was tested in all paradigms but was not repeat tested in any task. Each behavior was video recorded with the exception of the light/dark chamber, which was scored in real time. DVD (digital video disc)-recorded material was converted to MPEG (moving picture experts group) format using VideoWave Professional (Roxio, a division of Sonic Solutions) and then analyzed by a trained technician blind to all treatments using The Observer (Noldus Information Technologies). Behavioral data from each social test were analyzed using a two-way ANOVA (sex by siRNA treatment) with repeated measures as necessary in the sociability task (chamber durations as a within-subjects variable). Student-Newman-Keuls or Tukey's post hoc comparison tests were done based on significant findings in ANOVA tests. Behavioral data for anxiety tests for each sex were analyzed separately using Student's $t$ tests, because of the hormone manipulation necessary for standardized female anxiety-like behavior measurements. Mecp2 knockdown efficacy by Mecp2 siRNA versus control siRNA was analyzed with a Student's $t$ test. All reported measures are listed as mean \pm SEM.

Play behavior. The social play behavior paradigm was adapted from previously reported methods (Meaney and McEwen, 1986; Casto et al., 2003; Olesen et al., 2005). Animals were weaned on P21 and housed in groups of six, containing animals from each treatment condition (male control, male Mecp2 knockdown, female control, and female Mecp2 knockdown) and two (male and female) unrelated age-matched animals. Animals were tailmarked and video recorded in their home cages twice per day ( 1 and $3 \mathrm{~h}$ after lights off) for 4 min trials over $5 \mathrm{~d}$ (P25-P29) for a total observation time of $40 \mathrm{~min}$ per animal. Behavior was scored by an observer blind to the treatment groups. The play behavior scores were calculated by summing each animal's play-initiating behaviors (biting, chasing, pouncing, and pinning) over the entire observation time; group means were calculated based on summed individual total play initiating behaviors. Play behaviors were scored using the following criteria adapted from previously reported methods (Meaney and McEwen, 1986; Casto et al., 2003): (1) biting: one rat biting another; (2) chasing: one rat chases another; (3) pouncing: one rat pounces or lunges at another; and (4) pinning: one rat standing over another, with its forepaws on the ventral surface of the opposing rat.

Sociability. Sociability was measured with an adaptation of the social approach task (Crawley, 2004, 2007). An apparatus was split into three equally sized chambers with dividers that had sliding doors. Test animals were housed singly for $18 \mathrm{~h}$ and then placed in the center chamber for a 5 min acclimation period. After these $5 \mathrm{~min}$, social and nonsocial stimuli were placed in opposing outside chambers, and the dividing doors were opened, allowing the test animal to move freely between all chambers. The social stimulus was an age- and sex-matched novel rat held in a perforated plastic container $(7 \times 7 \times 16 \mathrm{~cm})$ that permitted sight, sound, scent, and minimal tactile contact. The nonsocial stimulus was an empty identical perforated plastic container. The stimulus animal had been habituated to this container and generally remained inactive during the test. Housing the stimulus in this container controlled the social interactions such that interactions were only initiated by the test animal. Time spent in each chamber and the numbers of crossings into each chamber were recorded for $10 \mathrm{~min}$.

Anxiety-like behavior tests and female preparation for testing. Under red light, $2 \mathrm{~h}$ after the dark phase of the light cycle began, animals were run through a series of three 5 min tests, including an elevated plus maze followed by a light/dark chamber and finishing in an open field arena. 
Each animal (age 60-65 d) was tested in this order of paradigms, and no repeat testing was performed. Because estrogens can modulate anxiety behavior, it was necessary to control peripheral estrogen release, which could vary significantly between animals during the estrus cycle. All females were ovariectomized and allowed to recover for $7 \mathrm{~d}$. Endogenous estrogen should clear during this time; this allowed us to control total estrogen with a peripheral injection. Each female was given $10 \mu \mathrm{g}$ of estradiol $48 \mathrm{~h}$ before behavioral testing.

Elevated plus maze. The elevated plus maze is a Plexiglas structure standing $50 \mathrm{~cm}$ off the floor and consisting of two opposing $100 \mathrm{~cm}$ runways that cross at the center. One runway is open, whereas the other is closed with 39-cm-high Plexiglas walls with an opening in the center to allow the other runway to cross; this opening is referred to as the "center." Rats were placed in the center of the maze facing an open arm; maze exploration was recorded for $5 \mathrm{~min}$. Parameters quantified were entries into as well as time spent in the open and closed arms and the center chamber. An entry was counted when all four paws crossed into a certain portion of the maze.

Light/dark chamber. The light/dark chamber is one large Plexiglas chamber split by an opaque Plexiglas insert into two compartments: one large lit side $(35 \times 38 \times 39 \mathrm{~cm})$ and one smaller dark side $(25 \times 38 \times 39$ $\mathrm{cm})$. An opening in the lower corner of the Plexiglas insert $(6 \times 10 \mathrm{~cm})$ allows the animal to move freely between the light and dark chambers. The dark side of the chamber is entirely constructed of opaque Plexiglas and equipped with an opaque lid. A white incandescent light is situated above the other chamber (light side), which is constructed of clear Plexiglas. The animal was placed in the middle of the light side of the box facing the opening and was observed for $5 \mathrm{~min}$. A cross from one compartment to the other was recorded when all four paws were on one side. Total time in the light side was recorded.

Open field test. Rats were placed into a corner of an $80 \mathrm{~cm}$ square arena with opaque walls $40 \mathrm{~cm}$ high and allowed to move freely for $5 \mathrm{~min}$. The arena floor had markings that divided it into 25 equal square $(16 \times 16$ $\mathrm{cm}$ ) divisions. The rat's movement in the arena was scored as the number of squares traveled during the 5 min testing period.

\section{Results}

\section{Mecp2 disruption with siRNA}

Animals were bilaterally infused with $100 \mathrm{nmol}$ of Mecp 2 or control siRNA. Confirmations of amygdala targeting were accomplished by infusing $1 \mu \mathrm{l}$ of a dye followed by rapid brain dissection to determine the site of infusion. Mecp 2 protein knockdown was confirmed for each sex ( $n \geq 6$ for each group, control and Mecp2 siRNA) by Western immunoblotting of homogenized, microdissected amygdala regions that had been infused on 3 consecutive days ( $\mathrm{P} 0-\mathrm{P} 2)$ and dissected $6 \mathrm{~h}$ past the final infusion (Fig. 1a,b). Mecp2 protein knockdown averaged $30-65 \%$, with greater siRNA effect in females, which may be related to higher basal expression of Mecp2 in female amygdala during this period of development. There was no effect of Mecp2 siRNA in adjacent hypothalamic sections that were collected with siRNA-targeted amygdala regions, which indicates the regional specificity of Mecp2 knockdown and control of siRNA spread (Fig. 1c). The transient disruption of Mecp2 was also confirmed in a separate experiment by Western immunoblotting of homogenized, microdissected amygdala regions $7 \mathrm{~d}$ after the final infusion (Fig. $1 d$ ). Test animals received three infusions to maintain reduced expression over the first $3 \mathrm{~d}$ of postnatal life, after which Mecp 2 levels were no longer experimentally manipulated. Animals recovered rapidly after each infusion under the warming light.
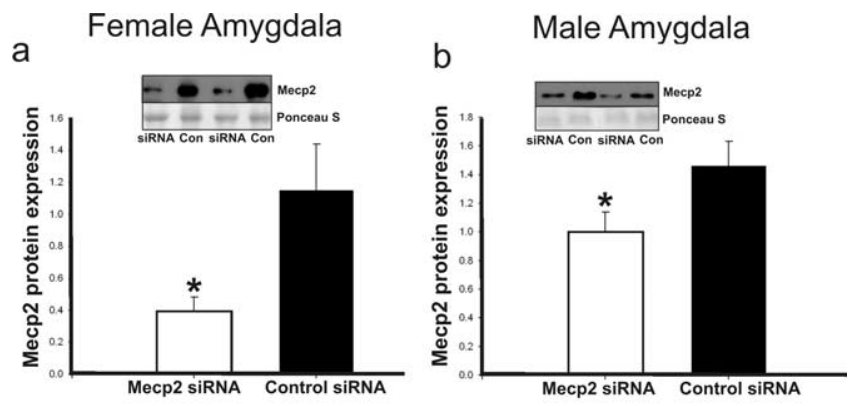

C
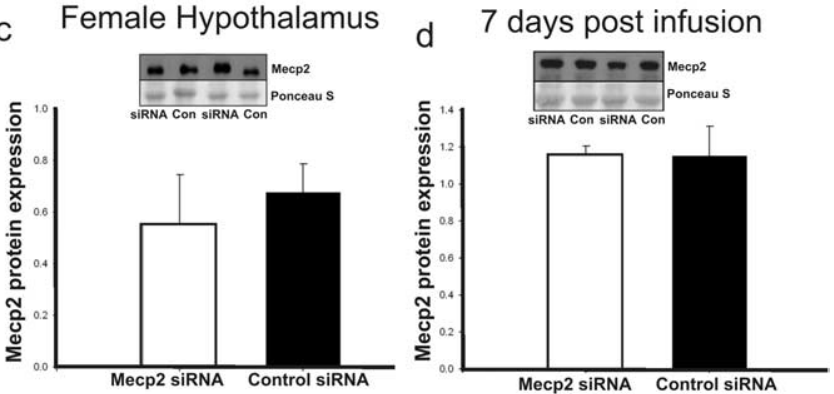

Figure 1. Mecp2 disruption with siRNA. Mecp2 siRNA effectively reduces Mecp2 protein expression at the site of infusion and allows recovery of typical Mecp2 expression with cessation of treatments. Bars represent means ( $n \geq 6$ for all groups in each panel), and error bars represent SEM. Each panel includes a representative immunoblot for Mecp2 protein expression above a corresponding Ponceau-S-stained band, which is used as a loading control. siRNA, Mecp2 siRNA; Con, control siRNA. $\boldsymbol{a}, \boldsymbol{b}$, Relative Mecp2 protein expression (Mecp2/Ponceau $S$ band optical density) in the amygdala, $6 \mathrm{~h}$ after the final Mecp2 or control siRNA infusions in females and males on P2, respectively. c, Relative Mecp2 expression in the hypothalamic sections adjacent to the amygdala region represented in $\boldsymbol{a}$. $\boldsymbol{d}$, Mecp2 relative expression in a separate experiment, in which male amygdala were analyzed for Mecp2 expression $7 \mathrm{~d}(\mathrm{P9})$ after three infusions on $\mathrm{PO}-\mathrm{P} 2$. This indicates that Mecp2 disruption was not manipulated by siRNA beyond this period of development. $\boldsymbol{a}$, Females: Mecp2 siRNA [0.396 \pm 0.085 (mean \pm SEM)]; control siRNA (1.149 \pm 0.029$) ;{ }^{*} p=0.02$. $\boldsymbol{b}$, Males: Mecp2 siRNA (1.000 \pm 0.137$)$; control siRNA $(1.460 \pm 0.172) ;{ }^{*} p=0.03$. c, Female hypothalamus: Mecp2 siRNA (0.557 \pm 0.188$)$; control siRNA (0.677 \pm 0.109$) ; p=0.592$. $\boldsymbol{d}$, Male time course: Mecp2 siRNA $(1.155 \pm 0.050)$; control $\operatorname{siRNA}(1.151 \pm 0.162) ; p=0.982$.

Neonatal treatment with Mecp2 siRNA within the developing amygdala disrupts sex differences in juvenile social play behavior

Our data indicate that typical Mecp2 expression within the developing amygdala of male rats is important for the organization of sex differences in juvenile behavior. Juvenile social play behavior in rats is commonly regarded as sexually dimorphic, with males engaging in this behavior more often than females (Meaney et al., 1983). We again report a sex difference in the initiation of play in the control infused animals (Fig. 2). This is an indication that our infusions did not significantly impair typical amygdala development, which is important for the organization of sexually dimorphic play behavior patterns (Meaney and Stewart, 1981; Meaney et al., 1981). Subtle Mecp2 disruption in females did not alter play behavior; however, male play behavior was significantly reduced in this condition (Fig. 2). Although Mecp2 disruption decreased male social play behavior, this reduction did not create an obvious hypoactive behavioral phenotype. Rather, these males exhibited levels of social play that resembled female typical play behavior. These data suggest that typical Mecp2 expression in males contributes to the sexually dimorphic pattern of social play commonly found in juvenile rats. 


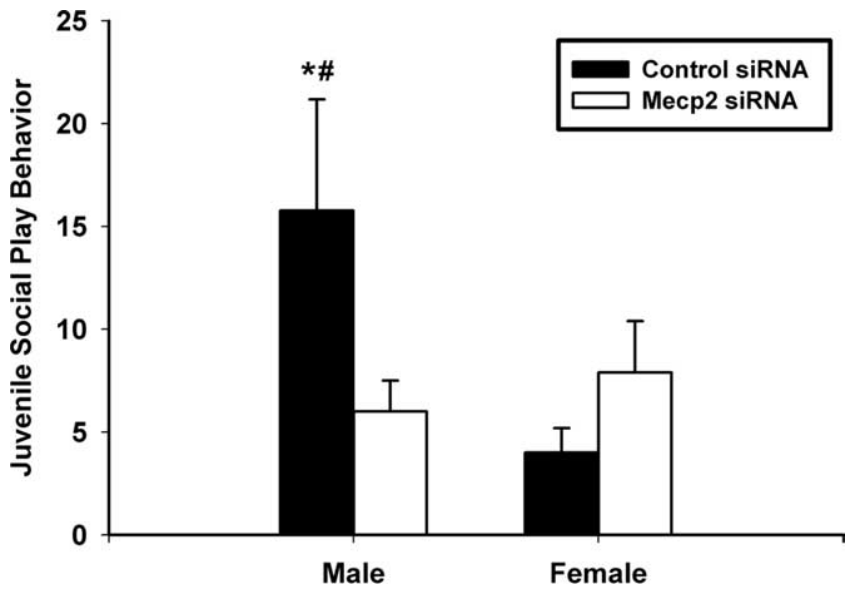

Figure 2. Mecp2 and sexual differentiation of juvenile play behavior. Typical Mecp2 expression is necessary for the patterning of sexually dimorphic juvenile (P25-P29) play behavior. Bars represent the group means ( $n \geq 8$ animals per group) of total initiated play behaviors within individuals. Error bars represent SEM. A significant interaction was observed (treatment by sex; two-way ANOVA; $p=0.048$ ) such that Mecp2 knockdown modified play behavior in males but not females. There were two main effects: (1) control males initiated play more often than control females [this is the typical sex difference in play behavior (Tukey's post hoc; ${ }^{*} p=$ 0.013)]; and (2) Mecp2 knockdown decreased play initiation within males (Tukey's post hoc; $\# p=0.037$ ) but did not change female play behavior (Tukey's post hoc; $p=0.484$ ). Control male, $15.78 \pm 3.18$ (mean \pm SEM); Mecp2 siRNA male, $6.00 \pm 3.17$; Control female, $4.00 \pm$ 3.18; Mecp2 siRNA female, $7.10 \pm 3.01$.

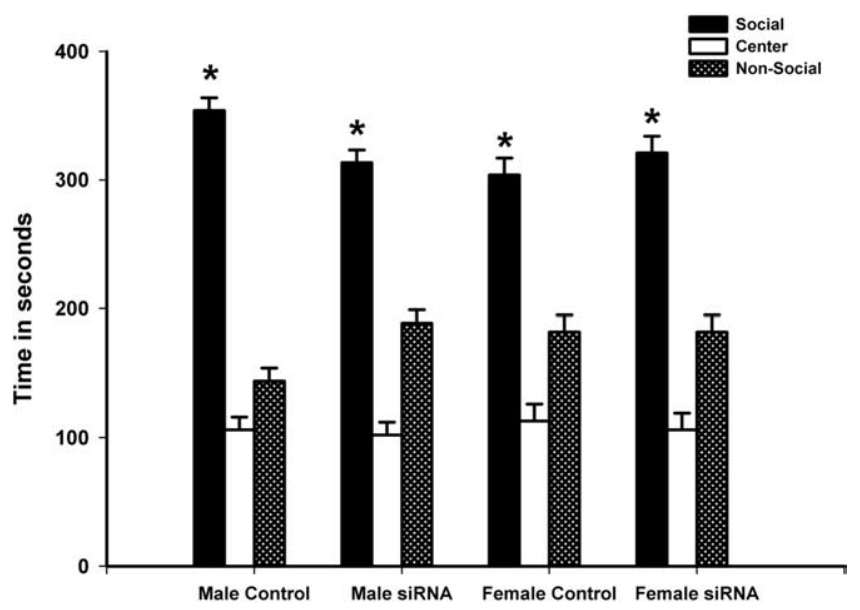

Figure 3. Mecp2 disruption and juvenile sociability. Transiently decreased Mecp2 expression in the developing amygdala did not disrupt sociability in juvenile (P31-P33) rats. All treatment groups showed normal levels of sociability, with time spent in the social chamber predominating over time spent in the nonsocial and middle chambers. Bars represent mean time ( $n \geq 8$ animals per group) spent in each chamber during a 10 min test, and error bars represent SEM. ${ }^{*} p<0.05$.

Neonatal treatment with Mecp2 siRNA within the developing amygdala does not disrupt juvenile sociability

All treatment groups showed normal levels of sociability, with time spent in the social chamber predominating over time spent in the nonsocial and middle chambers (Fig. 3): control males $(F=284.173 ; p<0.001)$, Mecp2 siRNA-treated males $(F=$ 78.72; $p<0.001)$, control females $(F=61.50 ; p<0.001)$, and Mecp 2 siRNA-treated females $(F=56.12 ; p<0.001)$. The total number of chamber crossings and side chamber entries serve as a means to evaluate general activity level. No differences were found for either measure between groups for sex ( $p \geq 0.3)$, treatment $(p \geq 0.1)$, or a sex by treatment interaction $(p \geq 0.8)$.
Table 1. Adult anxiety-like behavior

\begin{tabular}{llll}
\hline & $\begin{array}{l}\text { Elevated plus } \\
\text { maze }\end{array}$ & $\begin{array}{l}\text { Light/dark } \\
\text { chamber }\end{array}$ & $\begin{array}{l}\text { Open field } \\
\text { crosses }\end{array}$ \\
\hline $\begin{array}{l}\text { Males } \\
\text { Mecp2 siRNA }\end{array}$ & $37.0 \pm 8.9$ & $13.4 \pm 2.9$ & $54.3 \pm 12.8$ \\
$\begin{array}{c}\text { Control siRNA } \\
\text { Females }\end{array}$ & $40.0 \pm 9.7$ & $12.3 \pm 3.6$ & $60.1 \pm 15.8$ \\
Mecp2 siRNA & $37.0 \pm 8.7$ & $11.4 \pm 2.2$ & $66.8 \pm 12.5$ \\
Control siRNA & $36.7 \pm 10.9$ & $15.9 \pm 3.6$ & $70.5 \pm 15.7$ \\
\hline
\end{tabular}

No differences in adult (P60 - P65) anxiety-like behaviors were found with the brief neonatal disruption of amygdala Mecp2 expression. There was also no difference in locomotor activity in an open field arena. Elevated plus maze, Group means ( $n \geq 8$ animals per group for all tests) for total time spent (in seconds) on the open arms of the elevated plus maze during a 5 min test. Light-dark chamber, Group means for total time spent (in seconds) on the lit side of the light/dark chamber during a 5 min test. Open field crosses, Total number of square crosses in an open field arena. Data are presented as mean \pm SEM.

Chamber crossings were as follows: control male, $58.0 \pm 5.2$; Mecp2 male, $48.9 \pm 4.8$; control female, $52.4 \pm 4.6$; and Mecp2 female, $44.9 \pm 4.9$. Side chamber entries were as follows: control male, $29.0 \pm 2.6$; Mecp2 male, $24.4 \pm 2.4$; control female, $26.2 \pm$ 2.3; and Mecp2 female, $22.4 \pm 2.4$.

\section{Adult anxiety-like behaviors were not modified by neonatal amygdala Mecp2 disruption}

We determined the specificity of behavioral disruption by measuring anxiety-like behavior with the elevated plus maze and light/dark chamber. We found no differences in anxietylike behavior between conditions with either test (Table 1). Although these findings do not eliminate the potential relationship between increased anxiety and atypical male juvenile behavior, it is evident that adult anxiety-like behavior organization was not disturbed by short-term neonatal Mecp2 disruption as seen in male juvenile social behavior. Immediately after testing within the light/dark chamber, animals were placed in an open field arena for another test of locomotor activity. No differences were found in locomotion through the open field arena (Table 1).

\section{Discussion}

We reported previously that Mecp2 expression is sexually dimorphic in the developing rat brain, and this dimorphism exhibits temporal and regional patterns, with males expressing less Mecp2 protein within the amygdala and ventromedial hypothalamus during the first week of postnatal life (Kurian et al., 2007). Because reduced MeCP2 expression is suggested to occur in individuals with neurodevelopmental behavioral disorders with a male bias in diagnosis (Nagarajan et al., 2006), we questioned the susceptibility of male rats to behavioral modification after a brief developmental disruption of typical low Mecp2 expression. The use of siRNA enabled us to examine the effect of brief and subtle Mecp2 disruption within the developing amygdala and assess the enduring impact on juvenile social behavior organized by the amygdala. Unexpectedly, this disruption did not result in overt disordered behavior. We report that a subtle disruption of Mecp2 in the amygdala modified typical sex differences in juvenile social play behavior. Males engage in juvenile social play more often than females (Meaney et al., 1983). Mecp2 siRNA treatment reduced the frequency of juvenile social play behavior in males to female typical levels. Interestingly, females remained resilient to this disruption and exhibited no behavioral changes. Although neonatal treatment with Mecp2 siRNA dramatically altered the developmental organization of juvenile social play behavior in males, it did not disrupt juvenile sociability in males or females. These results suggest that male rats may be more sensitive to 
short-term subtle decreases in typical Mecp2 expression and that Mecp2 may be involved in the organization of sexually dimorphic social behavior.

Our results are surprising in that a subtle and brief disruption of Mecp2 in the amygdala eliminates a typical sex difference in juvenile social play behavior and suggest a novel role for this protein in sexually differentiating brain and behavior. Although our data indicate that Mecp2 siRNA reduced Mecp2 expression in both males and females, this disruption altered the development of juvenile social behavior only in males. It is not clear why Mecp2 siRNA females do not show a behavioral change in the social tasks examined. One possibility is that some genes on the $\mathrm{X}$ chromosome may exert a protective role during subtle decreases in Mecp2 expression during development, because X-linked genes may contribute to sex differences (De Vries et al., 2002; Gatewood et al., 2006; Xu and Disteche, 2006). Alternatively, because females typically express more Mecp2 protein than males at this time point, our data may suggest that a critical level of Mecp2 protein is required for typical social development that is organized in the amygdala. In this case, it is reasonable to conclude that tight and critical regulation of Mecp2 expression is important for social development and that males are particularly sensitive to Mecp2 disruption because they typically express less Mecp2 than females in this region and at this time point. A male sensitivity to Mecp2 disruption is an intriguing link to the observed male preponderance in neurodevelopmental social disorders associated with reduced MeCP2 expression. This link would suggest that understanding the regulation of Mecp2 expression or activity in the amygdala may provide evidence for factors that alter social development.

Because Mecp2 exhibits sexually dimorphic expression, which is restricted to a steroid-sensitive period for rat brain development (Kurian et al., 2007), it is an attractive hypothesis to implicate hormones in the regulation of Mecp2 expression. However, the factors that create this Mecp2 expression pattern remain unclear and complex, primarily because both hormones and nonhormonal pathways can contribute to sex differences in the brain (De Vries et al., 2002; Olesen et al., 2005). For example, whereas hormone manipulation during this period can eliminate the sex difference in juvenile play behavior, as found with Mecp2 disruption (Beatty et al., 1981; Meaney et al., 1983; Meaney and McEwen, 1986; Olesen et al., 2005), other factors such as modified dopamine levels or activity can also have a similar impact (Olesen et al., 2005). It is also possible that chromosomal factors can organize Mecp2 expression patterns independent of hormone levels (De Vries et al., 2002; Dewing et al., 2006). Clearly, multiple pathways may regulate Mecp2 expression, and clarification of the factors or combination of circumstances leading to altered Mecp2 expression should be considered with respect to their role in social behavior development.

Because MeCP2 can function through methyl-DNA binding (Nan et al., 1998), the functional role of this protein in social organization may also be dictated by indirect circumstances. For example, DNA methylation patterns are sensitive to social and environmental factors; this suggests the intriguing possibility that Mecp2-controlled gene expression may be influenced, in part, by social and environmental stimuli (Weaver et al., 2004). It remains to be fully elucidated how methylation patterns direct Mecp2 activity; however, distinguishing molecular or environmentally dictated differences in DNA methylation and their relationship to Mecp 2 activity may provide insight into the factors that participate in the organization of typical social behavioral patterns in males. Furthermore, because Mecp2 disruption eliminates the generation of typical sex differences in social behavior, these environmental factors may be considered potential epigenetic contributors to the development or exacerbation of sexually dimorphic behavior patterns.

The present understanding of an association between an epigenetic protein and sexually dimorphic social behavior development is intriguing and underscores the importance of future work exploring the concept of the epigenetic determination of sex differences in behavior. This work may offer invaluable information regarding circumstances leading to the manifestation of gender and consequently provide insights for points of divergence in the development and presentation of sex-biased behavioral disorders.

\section{References}

Amir RE, Van den Veyver IB, Wan M, Tran CQ, Francke U, Zoghbi HY (1999) Rett syndrome is caused by mutations in X-linked MECP2, encoding methyl-CpG-binding protein 2. Nat Genet 23:185-188.

Beatty WW, Dodge AM, Traylor KL, Meaney MJ (1981) Temporal boundary of the sensitive period for hormonal organization of social play in juvenile rats. Physiol Behav 26:241-243.

Casto JM, Ward OB, Bartke A (2003) Play, copulation, anatomy, and testosterone in gonadally intact male rats prenatally exposed to flutamide. Physiol Behav 79:633-641.

Chahrour M, Zoghbi HY (2007) The story of Rett syndrome: from clinic to neurobiology. Neuron 56:422-437.

Crawley JN (2004) Designing mouse behavioral tasks relevant to autisticlike behaviors. Ment Retard Dev Disabil Res Rev 10:248-258.

Crawley JN (2007) Mouse behavioral assays relevant to the symptoms of autism. Brain Pathol 17:448-459.

De Vries GJ, Rissman EF, Simerly RB, Yang LY, Scordalakes EM, Auger CJ, Swain A, Lovell-Badge R, Burgoyne PS, Arnold AP (2002) A model system for study of sex chromosome effects on sexually dimorphic neural and behavioral traits. J Neurosci 22:9005-9014.

Dewing P, Chiang CW, Sinchak K, Sim H, Fernagut PO, Kelly S, Chesselet MF, Micevych PE, Albrecht KH, Harley VR, Vilain E (2006) Direct regulation of adult brain function by the male-specific factor SRY. Curr Biol 16:415-420.

Funabashi T, Brooks PJ, Weesner GD, Pfaff DW (1994) Luteinizing hormone-releasing hormone receptor messenger ribonucleic acid expression in the rat pituitary during lactation and the estrous cycle. J Neuroendocrinol 6:261-266.

Gatewood JD, Wills A, Shetty S, Xu J, Arnold AP, Burgoyne PS, Rissman EF (2006) Sex chromosome complement and gonadal sex influence aggressive and parental behaviors in mice. J Neurosci 26:2335-2342.

Klein D, Kern RM, Sokol RZ (1995) A method for quantification and correction of proteins after transfer to immobilization membranes. Biochem Mol Biol Int 36:59-66.

Kurian JR, Forbes-Lorman RM, Auger AP (2007) Sex difference in mecp2 expression during a critical period of rat brain development. Epigenetics 2:173-178

Matsumoto A (1997) Hormonally induced neuronal plasticity in the adult motoneurons. Brain Res Bull 44:539-547.

Meaney MJ, McEwen BS (1986) Testosterone implants into the amygdala during the neonatal period masculinize the social play of juvenile female rats. Brain Res 398:324-328.

Meaney MJ, Stewart J (1981) Neonatal-androgens influence the social play of prepubescent rats. Horm Behav 15:197-213.

Meaney MJ, Dodge AM, Beatty WW (1981) Sex-dependent effects of amygdaloid lesions on the social play of prepubertal rats. Physiol Behav 26:467-472.

Meaney MJ, Stewart J, Poulin P, McEwen BS (1983) Sexual differentiation of social play in rat pups is mediated by the neonatal androgen-receptor system. Neuroendocrinology 37:85-90.

Nagai K, Miyake K, Kubota T (2005) A transcriptional repressor MeCP2 causing Rett syndrome is expressed in embryonic non-neuronal cells and controls their growth. Brain Res Dev Brain Res 157:103-106. 
Nagarajan RP, Hogart AR, Gwye Y, Martin MR, LaSalle JM (2006) Reduced $\mathrm{MeCP} 2$ expression is frequent in autism frontal cortex and correlates with aberrant MECP2 promoter methylation. Epigenetics 1:e1-e11.

Nan X, Ng HH, Johnson CA, Laherty CD, Turner BM, Eisenman RN, Bird A (1998) Transcriptional repression by the methyl-CpG-binding protein MeCP2 involves a histone deacetylase complex. Nature 393:386-389.

Olesen KM, Jessen HM, Auger CJ, Auger AP (2005) Dopaminergic activation of estrogen receptors in neonatal brain alters progestin receptor expression and juvenile social play behavior. Endocrinology 146:3705-3712.

Perrot-Sinal TS, Davis AM, McCarthy MM (2001) Developmental sex differences in glutamic acid decarboxylase $(\mathrm{GAD}(65))$ and the housekeeping gene, GAPDH. Brain Res 922:201-208.

Samaco RC, Hogart A, LaSalle JM (2005) Epigenetic overlap in autismspectrum neurodevelopmental disorders: MECP2 deficiency causes reduced expression of UBE3A and GABRB3. Hum Mol Genet 14:483-492.

Shibayama A, Cook EH Jr, Feng J, Glanzmann C, Yan J, Craddock N, Jones IR, Goldman D, Heston LL, Sommer SS (2004) MECP2 structural and 3'UTR variants in schizophrenia, autism and other psychiatric diseases: a possible association with autism. Am J Med Genet B Neuropsychiatr Genet 128B:50-53.

Stanley HF, Fink G (1986) Synthesis of specific brain proteins is influenced by testosterone at mRNA level in the neonatal rat. Brain Res 370:223-231.

Villard L (2007) MECP2 mutations in males. J Med Genet 44:417-423.

Weaver IC, Cervoni N, Champagne FA, D'Alessio AC, Sharma S, Seckl JR, Dymov S, Szyf M, Meaney MJ (2004) Epigenetic programming by maternal behavior. Nat Neurosci 7:847-854.

$\mathrm{Xu}$ J, Disteche CM (2006) Sex differences in brain expression of X- and Y-linked genes. Brain Res 1126:50-55. 